\title{
Design of Diversity-Achieving LDPC Codes for H-ARQ with Cross-Packet Channel Coding
}

\author{
Dieter Duyck \\ Ghent University \\ Gent, Belgium \\ dduyck@telin.ugent.be
}

\author{
Daniele Capirone \\ Politecnico di Torino \\ Torino, Italy \\ daniele.capirone@polito.it
}

\author{
Christoph Hausl \\ Technische Universität München \\ München, Germany \\ christoph.hausl@tum.de
}

\author{
Marc Moeneclaey \\ Ghent University \\ Gent, Belgium \\ mm@telin.ugent.be
}

\begin{abstract}
In wireless scenarios an effective protocol to increase the reliability for time-varying channels is the hybrid automatic repeat request (H-ARQ). The H-ARQ scheme with cross-packet channel coding (CPC) is a recently published extension of H-ARQ with several advantages. No full-diversity low-density paritycheck (LDPC) code design for the whole range of coding rates yielding full-diversity has been published. In this paper the authors provide a new outage behavior analysis and a new structured LDPC code ensemble achieving full-diversity for $\mathbf{H}$ ARQ with CPC by exploiting the rootcheck principle. Simulation results show that the new code design outperforms the previous approaches, providing full-diversity and good coding gain, also at high coding rates.
\end{abstract}

\section{INTRODUCTION}

Mobile communication systems have to cope with a timevarying signal-to-noise ratio (SNR) due to the effects of multipath and shadowing. If the instantaneous received signal strength is not known at the transmitter, high transmit power is needed to achieve low error rates with forward error correction (FEC). A simple and robust mechanism to achieve low error rates for time-varying channels is to use automatic repeat request $(A R Q)$ strategies [1], where the receiver asks for a retransmission $(\operatorname{ReTx})$ when it is not possible to decode the initial transmission (ITx).

Hybrid ARQ/FEC (H-ARQ) allows to combine the advantages of both ARQ and FEC [2]. In conventional H-ARQ schemes, the receiver combines the received values from ITx and ReTx, where ReTx contains incremental redundancy on the information sent in ITx. The error rate after ReTx is lower than after ITx because the transmission of incremental redundancy results in a lower coding rate and a better error protection. Furthermore, if the coherence time is short enough such that ReTx experiences another fading coefficient, diversity is gained.

H-ARQ with cross-packet channel coding (CPC) extends conventional H-ARQ schemes [3]. In this case, ReTx not only contains redundancy for the information sent in ITx but also contains new information. H-ARQ with CPC is more flexible to meet the requirements of applications. For example, in applications that require a constant rate, H-ARQ systems with CPC can transmit in every frame the same number of information bits and parity bits while still increasing the diversity order for previously transmitted packets that were not correctly decoded at the destination.
H-ARQ systems with CPC were designed based on turbo codes [3] and on LDPC codes [4], but these code designs do not guarantee full-diversity, especially if the coding rate approaches the maximum coding rate yielding full-diversity.

In this paper, we present the design of an improved crosspacket LDPC code whose structure guarantees to achieve full-diversity due to included rootcheck nodes [5]. Besides for point-to-point communication, the concept of rootchecks was also applied for relay channels [6], [7]. We show that our proposed LDPC code performs close to the information theoretical limit and outperforms the previous approaches in [3], [4] whose structures do not guarantee full-diversity.

The paper is structured as follows. In Section II the system model is described and conventional H-ARQ is compared to H-ARQ with cross-packet coding. In Section III, a new outage behavior analysis is given. Conclusions from this new outage behavior with respect to the achievable coding rates for a double diversity order are given in section IV. Section V elaborates on the diversity-achieving LDPC code construction.

\section{SySTEM MODEL}

In this section we will explain the difference between conventional H-ARQ and H-ARQ with CPC. As mentioned in the introduction, the contribution of this paper is the design of a full-diversity LDPC code construction for H-ARQ with $\mathrm{CPC}$. Because the choice of modulation does not alter the code structure, BPSK signaling is used.

\section{A. Conventional Hybrid ARQ/FEC}

The source receives information packets $\mathbf{u}_{i}$ of length $K$ from the application, where $i$ denotes the time index. A binary LDPC code $\mathcal{C}_{1}\left[N_{1}, K\right]$ with block length $N_{1}$, dimension $K$, and coding rate $R_{c 1}=K / N_{1}$ is used. The code $\mathcal{C}_{1}$ is defined by an $\left(N_{1}-K\right) \times N_{1}$ parity-check matrix $\mathbf{H}_{1}$, or equivalently, by the corresponding generator matrix $\mathbf{G}_{1}$ so that $\mathbf{G}_{1} \cdot \mathbf{H}_{1}^{T}=\mathbf{0}$, where $T$ denotes the transpose operation, $\cdot$ is a matrix multiplication, and $\mathbf{0}$ is the all-zero matrix. The source transmits $\mathbf{c}_{1, i}$ which can be expressed as

$$
\mathbf{c}_{1, i}=\mathbf{u}_{i} \cdot \mathbf{G}_{1} \text {. }
$$

Regardless of the adopted coding scheme an error detection system is embedded, for example with a cyclic redundancy check, which is attached to the packet $\mathbf{u}_{i}$ before encoding. 
If the receiver cannot successfully decode the information $\mathbf{u}_{i}$, a ReTx from the source is requested. As mentioned in the introduction, the receiver combines the received values of ITx and ReTx, which contains incremental redundancy on the information sent in ITx. This means that the destination decodes on one global error-correcting code $\mathcal{C}[N, K]$ with block length $N$, dimension $K$, and coding rate $R_{c}=K / N$. This code is defined by a parity-check matrix $\mathbf{H}$. In ReTx, the source transmits the remaining bits $\mathbf{c}_{2, i}$ of length $N_{2}$, $N_{1}+N_{2}=N$, which can be expressed as

$$
\mathbf{c}_{2, i}=\mathbf{u}_{i} \cdot \mathbf{G}_{2} .
$$

The generator matrix $\mathbf{G}_{2}$ corresponds to a parity-check matrix $\mathbf{H}_{2}$, so that $\mathbf{G}_{2} \cdot \mathbf{H}_{2}^{T}=\mathbf{0}$.

The next packet of information bits $\mathbf{u}_{i+1}$ is treated separately from the previous information packet $\mathbf{u}_{i}$. The above described transmitter is illustrated in Fig. 1.

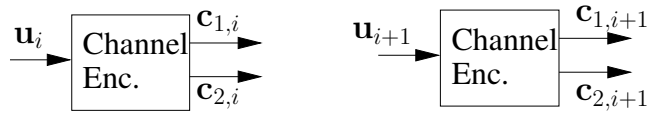

Figure 1. Transmission of two information packets $\mathbf{u}_{i}$ and $\mathbf{u}_{i+1}$ with conventional H-ARQ. If the receiver cannot decode $\mathbf{c}_{1, i}$, then $\mathbf{c}_{2, i}$ is transmitted. Every information packet is treated separately.

\section{B. Extension with Cross-Packet Channel Coding}

H-ARQ with CPC extends conventional ARQ in the sense that it allows the source to include $K_{2}$ additional information bits in its ReTx, which renders the protocol more flexible. By varying $K_{2}$, the number of additional information bits included in ReTx, we can scale the level of cross-packet channel coding. For $K_{2}=0$, the system falls back to conventional H-ARQ. By increasing $K_{2}$, the spectral efficiency increases as well. This can be practical in case the application requires a constant information rate for example. The level of cross-packet channel coding is described by the parameter $\sigma=K_{2} / K, \sigma \in[0, \infty]$.

Without loss of generality, we use a transmission scheme with $\sigma=1$, so that the source transmits an equal number of information bits $K$ in each transmission. The problem formulation is that until now, no near-outage (i.e. full-diversity and good coding gain) LDPC coding scheme has been published for all the achievable coding rates.

The information bit vector of length $K$ transmitted in ITx is denoted as $\mathbf{u}_{1, i}$. When the destination cannot decode ITx, a ReTx is requested and the information bit vector of length $K$ transmitted in ReTx is denoted as $\mathbf{u}_{2, i}$.

For ITx, there is no difference between conventional HARQ and H-ARQ with CPC, so that the transmitted code sequence $\mathbf{c}_{1, i}$ of length $N_{1}$ can be expressed as

$$
\mathbf{c}_{1, i}=\mathbf{u}_{1, i} \cdot \mathbf{G}_{1} .
$$

If the receiver cannot successfully decode the information $\mathbf{u}_{1, i}$, a ReTx from the source is requested. In contrast to conventional H-ARQ, the new transmitted code sequence $\mathbf{c}_{2, i}$ of length $N_{2}$ is a linear transformation of both $\mathbf{u}_{1, i}$ and $\mathbf{u}_{2, i}$ :

$$
\mathbf{c}_{2, i}=\left[\begin{array}{ll}
\mathbf{u}_{1, i} & \mathbf{u}_{2, i}
\end{array}\right] \cdot \mathbf{G}_{2} .
$$

This is illustrated in Fig. 2. The coding rate after ReTx is $R_{c}=\frac{2 K}{N}, N=N_{1}+N_{2}$.
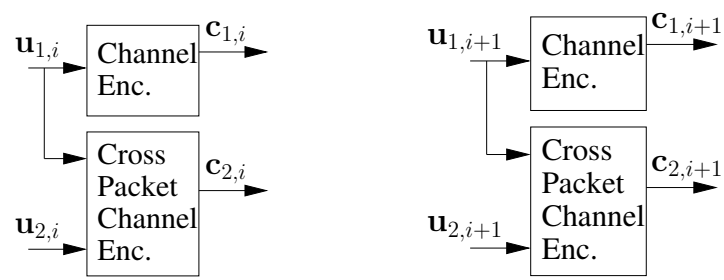

Figure 2. Transmission of two information packets $\mathbf{u}_{1, i}$ and $\mathbf{u}_{1, i+1}$ with $\mathrm{H}$-ARQ with CPC. If the receiver cannot decode $\mathbf{c}_{1, i}$, then $\mathbf{c}_{2, i}$ is transmitted. Here, $\mathbf{c}_{2, i}$ contains new information $\mathbf{u}_{2, i}$, so that the transmission rate can be easily tuned.

Using the principles explained in section $\mathrm{V}$, the proposed code structure can be extended to more ReTxs, as will be shortly discussed at the end of section V.

In this paper, the two scenarios that will be studied are summarized in Table I.

\begin{tabular}{|c|c|c|}
\hline & Case 1 & Case 2 \\
\hline$K$ & 1500 & 1500 \\
$N_{1}$ & 3000 & 1750 \\
$N_{2}$ & 3000 & 3000 \\
$R_{c 1}$ & $1 / 2$ & $6 / 7$ \\
$R_{c}$ & $1 / 2$ & $12 / 19$ \\
\hline
\end{tabular}

Table I

CODE PARAMETERS

\section{Channel Model}

We assume a noisy block fading (BF) channel model [8]. The fading coefficient of ITx is termed $\alpha_{1}$. When the destination cannot decode ITx, a ReTx is requested. The fading coefficient of this $\operatorname{ReTx}$ is termed $\alpha_{2}$, where $\alpha_{1}$ and $\alpha_{2}$ are i.i.d. Rayleigh distributed. The instantaneous signal-to-noise ratios of ITx and ReTx are given by $\gamma_{1}=\left|\alpha_{1}\right|^{2} \gamma$ and $\gamma_{2}=\left|\alpha_{2}\right|^{2} \gamma$ respectively, where $\gamma=E_{s} / N_{0}$ is the average symbol energy-to-noise ratio.

\section{OUtAGE BeHAVior}

The BF channel has a Shannon capacity that is essentially zero since the fading gain makes the mutual information a random variable which does not allow us to make the word error probability arbitrarily small under a certain spectral efficiency. The probability that mutual information be less than the transmitted rate ${ }^{1}$ is called information outage probability. The outage probability limit $p_{\text {out }}(\gamma)$ is a fundamental lower bound on the word error rate (WER) of coded communications systems [8].

\footnotetext{
${ }^{1}$ Because we use BPSK signaling, the transmitted spectral efficiency is equal to the coding rate.
} 


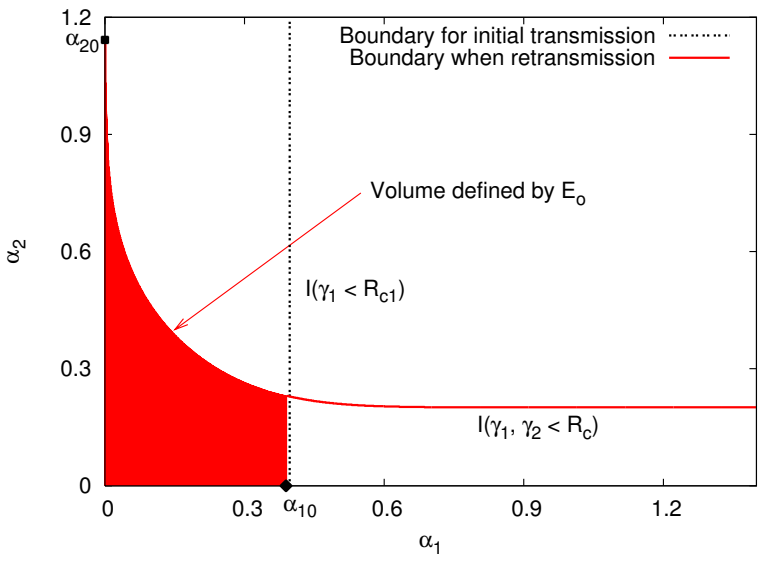

Figure 3. Illustration of the volume defined by an outage event $E_{o}$ in case 2 (see Table I). The average signal-to-noise ratio $\gamma=10 \mathrm{~dB}$. The points $\alpha_{10}$ and $\alpha_{20}$ are defined in Def. 2 .

Definition 1: The intrinsic diversity attained by a protocol is defined as

$$
d_{\text {out }}=-\lim _{\gamma \rightarrow \infty} \frac{\log p_{\text {out }}(\gamma)}{\log \gamma} .
$$

Here, we consider the outage behaviour for H-ARQ crosspacket channel coding. Given the channel realizations, an outage event $E_{o}$ indicates that the instantaneous mutual information be less than the transmitted rate. The outage event $E_{o}$ of this protocol is determined by a specific region in the twodimensional real space of instantaneous signal-to-noise ratios $\gamma_{1}$ and $\gamma_{2}$. Given a certain average $\operatorname{SNR} \gamma$, this corresponds to a specific region in the two-dimensional real space of fading gains $\alpha_{1}$ and $\alpha_{2}$. For H-ARQ with CPC, the outage event $E_{o}$ can be expressed as follows:

$$
E_{o}=\left(I_{1}\left(\gamma_{1}\right)<R_{c 1}\right) \cap\left(I_{2}\left(\gamma_{1}, \gamma_{2}\right)<R_{c}\right) .
$$

$I_{1}\left(\gamma_{1}\right)$ is the instantaneous mutual information of a point-topoint channel with a discrete input and instantaneous signal-tonoise ratio $\gamma_{1}, I_{1}\left(\gamma_{1}\right) \in[0,1]$ in the case of BPSK signaling. It is well known [9] how it has to be calculated. When $I_{1}\left(\gamma_{1}\right)<$ $R_{c 1}$, the mutual information is smaller than the transmitted rate in ITx, so that a ReTx is requested. ITx and ReTx behave as parallel channels whose capacities add together. Of course, both frames timeshare a time-interval, which gives a weight to each capacity term [10, Section 5.4.4]. Therefore, $I_{2}\left(\gamma_{1}, \gamma_{2}\right)$ can be expressed as follows:

$$
I_{2}\left(\gamma_{1}, \gamma_{2}\right)=\frac{N_{1}}{N} I_{1}\left(\gamma_{1}\right)+\frac{N_{2}}{N} I_{1}\left(\gamma_{2}\right) .
$$

Given Eqs. (5) and (6), we can obtain the volume defined by $E_{o}$ (Fig. 3 ). The outage probability $p_{\text {out }}(\gamma)$ is obtained by integrating the joint probability distribution $p\left(\alpha_{1}, \alpha_{2}\right)$ over the volume defined by $E_{o}$ :

$$
p_{\text {out }}(\gamma)=\iint_{E_{o}} p\left(\alpha_{1}, \alpha_{2}\right) \mathrm{d} \alpha_{1} \mathrm{~d} \alpha_{2} .
$$

We say that the volume $E_{o}$ is limited by an outage boundary $B_{o}\left(E_{o}\right)$.
Definition 2: We define $\alpha_{20}$ by the intersection between the outage boundary $B_{o}\left(E_{o}\right)$ and the axis $\alpha_{1}=0$. Next, $\alpha_{10}$ is the intersection between $B_{o}\left(E_{o}\right)$ and the axis $\alpha_{2}=0$.

Let us find an expression for $\alpha_{20}$ and $\alpha_{10}$. By definition, $I_{2}\left(0, \alpha_{20}^{2} \gamma\right)=\frac{N_{2}}{N} I_{1}\left(\alpha_{20}^{2} \gamma\right)=R_{c}$ and $I_{1}\left(\alpha_{10}^{2} \gamma\right)=R_{c 1}$ so that

$$
\begin{aligned}
\alpha_{10}^{2} & =\frac{I_{1}^{-1}\left(R_{c 1}\right)}{\gamma} \\
\alpha_{20}^{2} & =\frac{I_{1}^{-1}\left(R_{c} \frac{N}{N_{2}}\right)}{\gamma} .
\end{aligned}
$$

The argument of the function $I_{1}^{-1}($.$) is limited to 1$, because $I_{1}($.$) is the instantaneous mutual information of a point-to-$ point channel with a discrete BPSK input. Therefore, the conditions such that $\alpha_{20}$ and $\alpha_{10}$ belong to [0, $\infty$ are:

$$
\begin{aligned}
R_{c 1} & <1, \\
R_{c} & <\frac{N_{2}}{N} .
\end{aligned}
$$

Eq. (10) yields $N_{1}>K$ or $N_{2}<N-K$, so that

$$
R_{c}<1-\frac{K}{N}
$$

Using $\sigma=K_{2} / K$, (12) can be expressed as

$$
R_{c}<1-\frac{R_{c}}{\sigma+1}
$$

so that

$$
R_{c}<\frac{1+\sigma}{2+\sigma} .
$$

Definition 3: Outage boundary $B_{1}=B_{o}\left(E_{o}^{1}\right)$ is said to upperbound outage boundary $B_{2}=B_{o}\left(E_{o}^{2}\right)$ if $E_{o}^{2} \subset E_{o}^{1}$.

Proposition 1: In H-ARQ with CPC over a BF channel, the outage boundary $B_{o}\left(E_{o}\right)$ is upperbounded by the volume $E_{o}^{2}$ bounded by the lines $\alpha_{2}=\alpha_{20}$ and $\alpha_{1}=\alpha_{10}$.

Proof: A point $\left(\alpha_{1}, \alpha_{2}\right)$ in the fading space does not belong to the volume defined by $E_{o}$ if and only if $\left(I_{1}\left(\gamma_{1}\right) \geq R_{c 1}\right)$ or $\left(I_{2}\left(\gamma_{1}, \gamma_{2}\right) \geq R_{c}\right)$.

By definition, $I_{2}\left(0, \alpha_{20}^{2} \gamma\right)=R_{c}$. Because $I_{1}\left(\gamma_{1}\right) \geq 0$ [10], it can be easily proven with (6) that the mutual information $I_{2}\left(\alpha_{1}^{2} \gamma, \alpha_{20}^{2} \gamma\right) \geq I_{2}\left(0, \alpha_{20}^{2} \gamma\right)$ for $\alpha_{1}>0$.

Secondly, by definition $I_{1}\left(\alpha_{10}^{2} \gamma\right)=R_{c 1}$.

Therefore, the set of points defined by the lines $\alpha_{2}=\alpha_{20}$ and $\alpha_{1}=\alpha_{10}$ do not belong to the volume defined by $E_{o}$, because $I_{1}\left(\alpha_{1}^{2} \gamma\right) \geq R_{c 1}$ or $I_{2}\left(\alpha_{1}^{2} \gamma, \alpha_{2}^{2} \gamma\right) \geq R_{c}$.

Prop. 1 will serve in the proof of Prop. 2.

\section{DIVERSITY FOR H-ARQ WITH CPC}

It has been proved [11] that full-diversity is not achieved if $R_{c}>\frac{1+\sigma}{2+\sigma}$. However, the new analysis in section III gives new insights and allows to prove that full-diversity is achieved when $R_{c}<\frac{1+\sigma}{2+\sigma}$ which will be done in this section. We will also interpret this result to understand why this is true.

Proposition 2: In $\mathrm{H}-\mathrm{ARQ}$ with $\mathrm{CPC}$ over a $\mathrm{BF}$ channel with $\sigma=K_{2} / K$, the combination of coding rates $R_{c}<\frac{1+\sigma}{2+\sigma}$ and $R_{c 1}<1$ yields an intrinsic diversity order $d_{\text {out }}=2$.

Proof: The outage probability $p_{\text {out }}$ is obtained by (7). Consider the volume bounded by the lines $\alpha_{2}=\alpha_{20}$ and 
$\alpha_{1}=\alpha_{10}$ if $\alpha_{20}$ and $\alpha_{10}$ exist. In Prop. 1, it is proved that $E_{o}$ is a subset of this volume, so that $p_{\text {out }}$ is upperbounded by

$$
p_{\text {out }}^{u p}=\int_{0}^{\alpha_{10}} \int_{0}^{\alpha_{20}} p\left(\alpha_{1}, \alpha_{2}\right) \mathrm{d} \alpha_{1} \mathrm{~d} \alpha_{2}
$$

Taking normalized i.i.d. Rayleigh distributed fading gains, $p\left(\alpha_{1}, \alpha_{2}\right)=4 \alpha_{1} \alpha_{2} e^{-\alpha_{1}^{2}-\alpha_{2}^{2}}$, so that

$$
p_{\text {out }}^{\text {up }}=\left(1-e^{-\alpha_{20}^{2}}\right)\left(1-e^{-\alpha_{10}^{2}}\right)
$$

For $\gamma \rightarrow \infty$, the exponentials can be approximated by their Taylor expansion, yielding

$$
p_{\text {out }}^{u p}=\alpha_{20}^{2} \alpha_{10}^{2}+O\left(\alpha_{20}^{2} \alpha_{10}^{2}\right)
$$

so that through Eqs. (8) and (9)

$$
p_{\text {out }}^{u p} \propto \frac{1}{\gamma^{2}} .
$$

The proof holds for $R_{c 1}<1$ and $R_{c}<\frac{1+\sigma}{2+\sigma}$ (see Eqs. (10) and (14)).

Proposition 3: In H-ARQ with CPC over a BF channel with cross-packet level $\sigma$, transmitting at a coding rate $R_{c}$ greater than $\frac{1+\sigma}{2+\sigma}$ renders a single order diversity.

Proof: It is sufficient to prove the stated proposition over a Block Binary Erasure Channel (block-BEC) [12], because it is an extremal case of a BF channel. In a block-BEC, the fading gains $\alpha_{1}$ and $\alpha_{2}$ take two possible values $\{0, \infty\}$, so that the mutual information $I_{1}($.$) takes two possible values \{0,1\}$. In the case that a $\operatorname{ReTx}$ is required $\left(I_{1}\left(\gamma_{1}=0\right)<R_{c 1}\right)$, an outage event is declared if $I_{2}\left(\gamma_{1}=0, \gamma_{2}=\infty\right)<R_{c}$ (see Eq. (5)). From (6), we know that $I_{2}\left(\gamma_{1}=0, \gamma_{2}=\infty\right)=\frac{N_{2}}{N}=\frac{1+\sigma}{2+\sigma}$, so that an outage event is declared if $R_{c}>\frac{1+\sigma}{2+\sigma}$, even if $\gamma_{2}=\infty$.

In this paper, $\sigma=1$ is taken so that the coding rate $R_{c}$ must not be greater than $2 / 3$. This results is remarkable because for a normal BF channel (no H-ARQ) with two channel states where also $2 K$ information bits are equally split on two fading gains, it is known that the maximum achievable coding rate is $R_{c}=0.5$ [13]. The difference however is that in normal $\mathrm{BF}$ channels, the outage boundary is not cut by the line $I_{1}\left(\gamma_{1}\right)<R_{c 1}$. If the outage boundary would not have been cut by this line, $\alpha_{10}$ would not exist and full-diversity would not be achieved (Fig. 3). The reason is that the set of $K$ information bits transmitted in ReTx would not be recovered if the second fading gain $\alpha_{2} \rightarrow 0$. This is because the number of parity bits transmitted in ITx is too low. However, for HARQ, these parity bits are less important because the set of $K$ information bits transmitted in ReTx is only sent when $\alpha_{1}$ is very low which reduces the importance of the parity bits transmitted in ITx. Furthermore, the fraction of the time that a ReTx occurs behaves as $\frac{1}{\gamma}$ so that the performance of the set of $K$ information bits transmitted in ReTx cannot determine the diversity order. This is clarified formally in the next section with Eq. (21). This is exactly what is illustrated by the cutting line $I_{1}\left(\gamma_{1}\right)<R_{c 1}$ in Fig. 3 .

\section{Diversity-Achieving LDPC Code Design}

In this section we design a diversity achieving LDPC code for H-ARQ with cross-packet channel coding in the case of only one ReTx. The code defines a unique mapping from the information packet $\mathbf{u}_{1, i}$ to the coded packet $\mathbf{c}_{1, i}$ and from the information packets $\mathbf{u}_{1, i}$ and $\mathbf{u}_{2, i}$ to the coded packet $\mathbf{c}_{2, i}$, as illustrated in Fig. 2.

Definition 4: The diversity order attained by a code $\mathcal{C}$ is defined as

$$
d=-\lim _{\gamma \rightarrow \infty} \frac{\log P_{e}}{\log \gamma}
$$

where $P_{e}$ is the word error rate after decoding.

Let us denote the number of erroneously decoded packets $\mathbf{u}_{1, i}$ and $\mathbf{u}_{2, i}$ by $E_{1}$ and $E_{2}$ respectively. The number of transmitted packets $\mathbf{c}_{1, i}$ and $\mathbf{c}_{2, i}$ is denoted by $T_{1}$ and $T_{2}$ respectively. For H-ARQ with CPC, the WER $P_{e}$ is defined as follows:

$$
P_{e}=\frac{E_{1}+E_{2}}{T_{1}+T_{2}}
$$

This can be expressed alternatively:

$$
P_{e}=\frac{E_{1}}{T_{1}} \frac{T_{1}}{T_{1}+T_{2}}+\frac{E_{2}}{T_{2}} \frac{T_{2}}{T_{1}+T_{2}}
$$

Where $\frac{T_{1}}{T_{1}+T_{2}}$ is the probability that ITx was successful, $\frac{T_{1}}{T_{1}+T_{2}}=\left(1-\frac{c}{\gamma}\right)$ where $c$ is a constant, and $\frac{T_{2}}{T_{1}+T_{2}}$ is the probability that ITx cannot be decoded, $\frac{T_{2}}{T_{1}+T_{2}} \propto \frac{1}{\gamma}$. Because $\frac{E_{2}}{T_{2}} \propto \frac{1}{\gamma^{d_{2}}}$, where $d_{2} \geq 1$,

$$
d=2 \Longleftrightarrow \frac{E_{1}}{T_{1}+T_{2}} \propto \frac{1}{\gamma^{2}} .
$$

Before going into details of the code design, it is convenient to consider the block-BEC. A code that has not full-diversity on a block-BEC does not have full-diversity on a BF channel. Furthermore, a code has full-diversity on a BF channel if and only if it has full-diversity on a block BEC [5]. This design rule is very powerful because with the assumption of a blockBEC channel it is easy to check whether the designed code has full-diversity or not. For example, given the diversity rule (21), the code must be able to recover all information packets $\mathbf{u}_{1, i}$ if $\alpha_{1}=0$ and $\alpha_{2}=\infty$. Note that (21) indicates that the code must not be able to recover all information packets $\mathbf{u}_{2, i}$ if $\alpha_{1}=\infty$ and $\alpha_{2}=0$, which takes into account the intrinsic asymmetry of the H-ARQ system ${ }^{2}$, which is also the reason why coding rates higher than $R_{c}=0.5$ are achievable, as discussed at the end of the previous section.

\section{A. Initial transmission}

During ITx a generic systematic LDPC code with a coding rate $R_{c 1}=K / N_{1}$ and a parity check matrix $\mathbf{H}_{1}$ is used. Because a systematic encoder is used, the $N_{1}$ coded bits sent in ITx are divided in two classes, an information packet of the class $u_{1}$ and a parity packet of the class $p_{1}$.

\footnotetext{
${ }^{2}$ If $\alpha_{1}=\infty$, the receiver is able to decode $\mathbf{u}_{1, i}$ and no ReTx would be requested, so that this scenario does not occur.
} 


\section{B. Retransmission}

The systematic form allows to easily design the second constituent code using the rootcheck principle [5] that ensures full-diversity for the information bits of the class $u_{1}$. We remind that full-diversity means that the code must be able to recover the information packets $\mathbf{u}_{1, i}$ if $\alpha_{1}=0$ and $\alpha_{2}=\infty$. In Fig. 4 a rootcheck node is represented: it is a special type of check node, where all the leaves are related to bits affected by a fading instance different from the root's. If the coded symbols are transmitted on a block-BEC channel such that the white leaves are perfectly received while the red root is erased, the message passing belief propagation algorithm can solve the one-unknown parity-check equation in one decoding iteration.

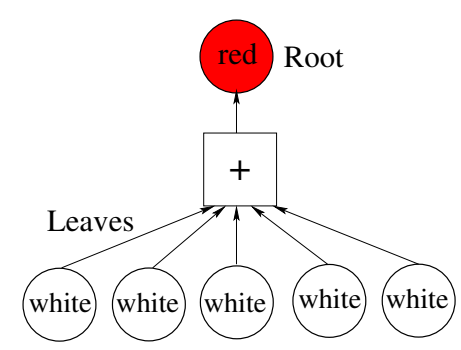

Figure 4. The rootcheck node can recover the erased red root bit if all the white leave bits are known.

A ReTx is required when ITx has been erased. The second constituent code, if not erased, has to be able to recover all the erased information bits from ITx. It encodes $\mathbf{u}_{1, i}$ and $\mathbf{u}_{2, i}$ ( $2 K$ information bits) and transmits $\mathbf{c}_{2, i}$ ( $N_{2}$ coded bits). We classify the bits transmitted in ReTx in the class $c_{2}$. The number of unknown is $K$, the number of erased information bits from ITx, so that the number of parity check equations of the second constituent code must be greater than or equal to $K$. This way, each of the information bits of the class $u_{1}$ can be protected by a rootcheck. Indeed, if ReTx is not erased (i.e., $\alpha_{2}=+\infty$ ) the $K$ information bits, $\mathbf{u}_{1, i}$, can be recovered in one message-passing decoding iteration. From this consideration the structure of the parity check matrix can be directly drawn as follows:

$$
\mathbf{H}=\left[\begin{array}{ccc}
u_{1} & p_{1} & c_{2} \\
\mathbf{H}_{1 i} & \mathbf{H}_{1 p} & \mathbf{0} \\
\mathbf{P} & \mathbf{0} & \mathbf{H}_{c 2}
\end{array}\right] \begin{gathered}
1 c \\
2 c
\end{gathered}
$$

where $\mathbf{P}$ is a permutation matrix and $1 c$ and $2 c$ indicate the sets of parity check equations of the first and second constituent code respectively. Alternatively, they are the corresponding parity-check matrices of the generator matrices $\mathbf{G}_{1}$ and $\mathbf{G}_{2}$ respectively. The first packet $\mathbf{u}_{1, i}$ is connected to both sets of parity check equations. The set of parity-check equations $2 c$ (containing the permutation matrix concatenated with the zero matrix) guarantee a double diversity order. The reason is that in the scenario where $\alpha_{1}=0$ and $\alpha_{2}=\infty$, the set of equations $2 c$ correspond to a set of $K$ equations where each equation has only one unknown. This set of equations can be easily solved by the sum-product algorithm. Using Props. 6 and 7 in [5], it can be easily proven that this code construction also has full-diversity on a BF channel.

The code structure in (22) is similar to the Root-LDPC structure [5] but it takes into account the intrinsic asymmetry of the H-ARQ system. For this reason we refer to it with the name H-ARQ Root-LDPC code.

\section{Extension to multiple retransmissions}

When more retransmissions are allowed, a higher intrinsic diversity order can be obtained. This can be achieved by LDPC codes by applying the rootcheck principle. Now, every information bit will be connected to multiple rootchecks [5]. For example, consider a system with two retransmissions. Each bit of the first packet of information bits $\mathbf{u}_{1, i}$ is connected to two rootchecks. Each bit of the second packet of information bits $\mathbf{u}_{2, i}$ is connected to one rootcheck (which is sufficient since the fraction of the time that one ReTx occurs behaves as $\frac{1}{\gamma}$ ). The bits of the third packet of information bits $\mathbf{u}_{3, i}$ are not connected to rootchecks because the fraction of the time that two ReTx occur behaves as $\frac{1}{\gamma^{2}}$. The parity-check matrix achieving a triple diversity order is a simple extension of (22).

$$
\mathbf{H}=\left[\begin{array}{ccccc}
u_{1} & p_{1} & u_{2} & p_{2} & c_{3} \\
\mathbf{H}_{1 i} & \mathbf{H}_{1 p} & \mathbf{0} & \mathbf{0} & \mathbf{0} \\
\mathbf{P} & \mathbf{0} & \mathbf{H}_{2 i} & \mathbf{H}_{2 p} & \mathbf{0} \\
\mathbf{P} & \mathbf{0} & \mathbf{P} & \mathbf{0} & \mathbf{H}_{c 3}
\end{array}\right] \begin{aligned}
& 1 c \\
& 2 c \\
& 3 c
\end{aligned}
$$

Because of the lack of space and because it is not the focus of the paper, this will be more elaborated in future work.

\section{Simulation Results}

In this section we compare the proposed code, designed for H-ARQ systems with cross-packet channel coding, to the coding schemes existing in literature.

We simulate the diversity achieving H-ARQ Root-LDPC code described in Section V and we measure the WER for BPSK as described in (19). We test two different scenarios that correspond to two different values of $R_{c 1}$ (see Table I).

In particular we compare the H-ARQ Root-LDPC performance with the following reference systems:

- Turbo-Turbo: turbo codes with the same coding rates are used in ITx and ReTx [3], [11].

- H-ARQ Random LDPC: an LDPC code defined by a parity check matrix whose form is represented in (24). In this article we use two different formulations proposed in literature which have the same performance: 1) $\mathbf{H}_{12}$ is a random matrix while $\mathbf{H}_{22}$ is an all-zero matrix. This code has been proposed and simulated in [4] for Case 1. 2) the sub-matrix $\left[\begin{array}{ll}\mathbf{H}_{12} & \mathbf{H}_{22}\end{array}\right]$ is a very sparse matrix with one ' 1 ' per column. This code is inspired by the rate compatible codes [14], where the code extension problem is exhaustively analyzed. In both cases $\mathbf{H}_{1}=\left[\begin{array}{ll}\mathbf{H}_{1 i} & \mathbf{H}_{1 p}\end{array}\right]$ and $\mathbf{H}_{c 2}$ are similar to the H-ARQ Root-LDPC code case.

$$
\mathbf{H}=\left[\begin{array}{lll}
\mathbf{H}_{1 i} & \mathbf{H}_{1 p} & \mathbf{0} \\
\mathbf{H}_{12} & \mathbf{H}_{22} & \mathbf{H}_{c 2}
\end{array}\right] \begin{aligned}
& 1 c \\
& 2 c
\end{aligned}
$$


To check the performance of the H-ARQ Root-LDPC code, $\mathbf{H}_{1}=\left[\begin{array}{ll}\mathbf{H}_{1 i} & \mathbf{H}_{1 p}\end{array}\right]$ and $\mathbf{H}_{c 2}$ must be generated. For each of these two matrices, consider the random ensemble of binary $(\lambda(x), \rho(x))$ LDPC codes (see Table II), where $\lambda(x)$ defines the left degree distribution and $\rho(x)$ defines the right degree distribution, both from a node perspective [2].

\begin{tabular}{|c|l|l|}
\hline Name & Left degrees & Right degrees \\
\hline \hline Case 1- $\mathbf{H}_{1}$ & $\lambda_{3}=1.0$ & $\rho_{6}=1.0$ \\
\hline Case 1- $\mathbf{H}_{c 2}$ & $\lambda_{3}=1.0$ & $\rho_{6}=1.0$ \\
\hline Case 2- $\mathbf{H}_{1}$ & $\lambda_{2}=0.4$ & $\rho_{23}=0.5$ \\
& $\lambda_{3}=0.22$ & $\rho_{24}=0.5$ \\
& $\lambda_{5}=0.38$ & \\
\hline Case 2- $\mathbf{H}_{c 2}$ & $\lambda_{3}=1.0$ & $\rho_{6}=1.0$ \\
\hline
\end{tabular}

Table II

Characteristics of $\mathbf{H}_{1}$ AND $\mathbf{H}_{c 2}$ IN CASE 1 AND CASE 2.

A better choice of the degree distributions will be studied in future work.

For the Turbo-Turbo codes, the UMTS parallel concatenated convolutional code is used [15]. All the performance curves are also compared to the outage probability as defined in Section III. Figs. 5 and 6 show the overall performance of the coding schemes in both scenarios. We notice that the H-ARQ RootLDPC code achieves full-diversity while the Random-LDPC code and the Turbo-Turbo code show a single order diversity.

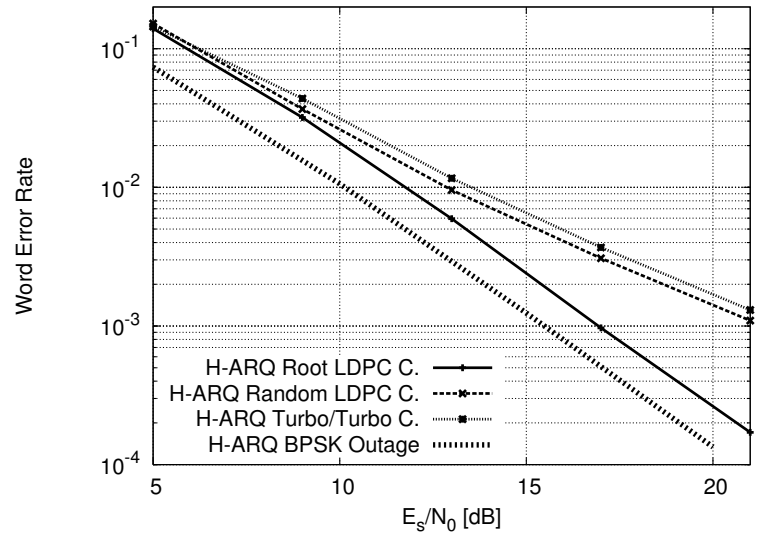

Figure 5. Case 1: word error rate performance of H-ARQ with CPC

\section{CONCLUSION}

We studied LDPC codes for H-ARQ with cross-packet channel coding systems and one ReTx in a slowly varying fading environment under iterative decoding. We presented a new outage behaviour analysis giving insights on the achievable diversity order. The authors proposed a new structured LDPC code family that achieves full-diversity. Simulation results showed that the new proposed code outperforms all the previous code designs.

A natural continuation of this research aims to improve the coding gain of the H-ARQ Root-LDPC code. Future works will also focus on the extension of the H-ARQ Root-LDPC code to multiple ReTxs.

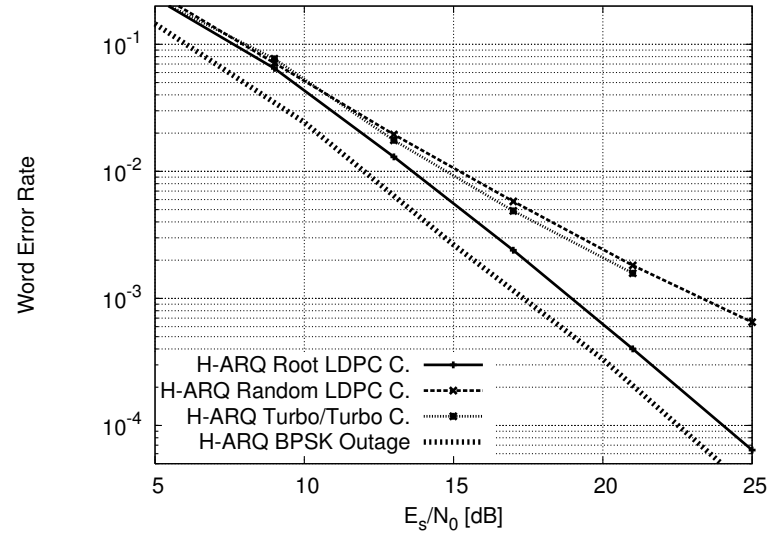

Figure 6. Case 2: word error rate performance of H-ARQ with CPC

\section{ACKNOWLEDGEMENT}

This work was supported by the European Commission in the framework of the FP7 Network of Excellence in Wireless COMmunications NEWCOM++ (contract n. 216715).

\section{REFERENCES}

[1] S. Lin, D. J. Costello, and M. J. Miller. Automatic repeat-request error control schemes. IEEE Communications Magazine, 12:5-17, Dec. 1984.

[2] S. Lin and D. J. Costello, Jr. Error Control Coding. Pearson Prentice Hall, 2004.

[3] C. Hausl and A. Chindapol. Hybrid arq with cross-packet channel coding. Communications Letters, IEEE, 11(5):434-436, May 2007.

[4] J. Chui and A. Chindapol. Design of cross-packet channel coding with low-density parity-check codes. In Information Theory for Wireless Networks, 2007 IEEE Information Theory Workshop on, pages 1-5, 1-6 July 2007.

[5] J.J. Boutros, A. Guillén i Fàbregas, E. Biglieri, and G. Zemor. LowDensity Parity-Check Codes for Nonergodic Block-Fading Channels. submitted to IEEE Trans. on Inf. Theory in Oct. 2007.

[6] D. Duyck, J.J. Boutros, and M. Moeneclaey. Low-Density Graph Codes for Slow Fading Relay Channels. submitted to IEEE Trans. on Inf. Theory in Mar. 2009.

[7] D. Duyck, D. Capirone, M. Moeneclaey, and J.J. Boutros. A fulldiversity joint network-channel code construction for cooperative communications. In International Symposium on Personal, Indoor and Mobile Radio Communications (PIMRC), Sept. 2009.

[8] E. Biglieri, J. Proakis, and S. Shamai. Fading channels: Informationtheoretic and communications aspects. IEEE Transactions on Information Theory, 44(6):2619-2692, 1998.

[9] G. Ungerboeck. Channel coding with multilevel/phase signals. IEEE Trans. Inform. Theory, IT-28(1):55-67, 1982.

[10] D. Tse and P. Viswanath. Fundamentals of Wireless Communication. Cambridge University Press, 2005.

[11] C. Hausl. Joint Network-Channel Coding for Wireless Relay Networks. Dissertation, Technische Universität München, Nov. 2008.

[12] A. Lapidoth. The performance of convolutional codes on the block erasure channel using various finite interleaving techniques. IEEE Trans. Inform. Theory, IT-40(5):1459-1473, Sep. 1994.

[13] R. Knopp and P. A. Humblet. On Coding for Block Fading Channels. IEEE Trans. Inform. Theory, 46(1):189-205, Jan. 2000.

[14] J. Li and K. Narayanan. Rate-compatible low density parity check codes for capacity-approaching ARQ scheme in packet data communications. In Int. Conf. on Comm., Internet, and Info. Tech.(CIIT), pages 201-206. Citeseer, 2002.

[15] M. C. Valenti and J. Sun. The UMTS Turbo Code and an Efficient Decoder Implementation Suitable for Software-Defined Radio. Intern Journal of Wireless Information Networks, 8(4):203-215, Oct. 2001. 